\title{
eJRIEPS
}

Ejournal de la recherche sur l'intervention en éducation physique et sport

45 | 2019

Varia

\section{Les situations émotionnellement marquantes en EPS : traits typiques et rôles dans le développement professionnel des enseignants novices}

\section{Magali Descoeudres et Jacques Méard}

\section{(2) OpenEdition}

Édition électronique

URL : http://journals.openedition.org/ejrieps/626

DOI : 10.4000/ejrieps.626

ISSN : 2105-0821

Éditeur

ELLIADD

\section{Référence électronique}

Magali Descoeudres et Jacques Méard, «Les situations émotionnellement marquantes en EPS : traits typiques et rôles dans le développement professionnel des enseignants novices », eJRIEPS [En ligne], 45 | 2019, mis en ligne le 01 avril 2019, consulté le 01 août 2019. URL : http://journals.openedition.org/ ejrieps/626 ; DOI : 10.4000/ejrieps.626

La revue eJRIEPS est mise à disposition selon les termes de la Creative Commons Attribution 4.0 International License. 


\section{Les situations émotionnellement marquantes en EPS : traits typiques et rôles dans le développement professionnel des enseignants novices}

Magali Descoeudres* \& Jacques Méard*

*UER d'éducation physique, Haute école pédagogique du canton de Vaud, Suisse.

Résumé

Cette recherche vise à identifier les types de situations émotionnellement marquantes vécues par les enseignants novices (EN) d'éducation physique et sportive (EPS) en classe. Un questionnaire qualitatif (demandant de rapporter deux situations émotionnellement marquantes) a été documenté par $139 \mathrm{EN}(\mathrm{N}=278)$ et traité de façon inductive. Les résultats montrent que ces situations sont regroupées en 12 types. Les EN en EPS rapportent des situations conduisant pour deux tiers à des émotions négatives, en lien notamment avec la transgression de règles par les élèves, la gestion de cas d'élèves particuliers et celles impliquant l'intégrité physique d'un élève. La plupart des EN déclarent que ces situations ont favorisé leur développement professionnel. De plus, ils ont partagé ces situations avec leur tuteur, leurs collègues, mais aussi leurs amis et conjoints dans des proportions importantes. Ces résultats sont discutés avec des éléments de la littérature et d'un point de vue développemental.

Mots-clés: Education physique - enseignants novices - développement - situation émotionnelle

\section{Introduction}

Le phénomène d'abandon du métier par les enseignants novices (EN) prend des proportions préoccupantes dans les systèmes scolaires occidentaux, notamment aux EtatsUnis (Burke, Schuck, Aubusson, Buchanan, Louvière \& Prescott, 2013 ; Craig, 2014; Craig, 2017). Ce "décrochage professionnel » précoce augmente également dans d'autres pays et les études qualitatives tendent à le mettre en rapport avec ce qui est décrit comme une surcharge émotionnelle en début de carrière (Harfitt, 2015 ; Visioli, Petiot \& Ria, 2015 ; Zimmermann \& Méard, 2016). 


\section{eJRIEPS 45 (Avril 2019)}

Les questionnements relatifs à la nature et aux effets des émotions ressenties par les enseignants dans l'exercice de leur métier ont été initiés dans le monde anglo-saxon par les travaux de Hargreaves $(1998,2000)$, relayés dans la sphère francophone par Visioli et Ria (2010), notamment à propos des enseignants débutants (Ria, Sève, Saury, Theureau, \& Durand, 2003). L'émotion, comme état mental dans une situation donnée, précède le sentiment et la prise de décision et se définit ici comme une « réponse publiquement observable » du fait de «marqueurs somatiques » (Damasio, 1994). Elle est aussi une manière de communiquer aux autres ses propres états mentaux. Dans ce cadre, la capacité pour l'enseignant à reconnaître les émotions chez autrui, à être capable de simuler ou de masquer ses propres émotions, relèverait donc d'un "construit" qui caractériserait l'expertise du professionnel (Chen, 2016 ; Visioli, Petiot \& Ria, 2015). Cette facette du métier impacte d'ailleurs le comportement des élèves (Chang, 2013) et leurs apprentissages (Brackett, Floman, Ashton-James, Cherkasskiy, \& Salovey, 2013). Ils ont un effet également sur l'activité des enseignants eux-mêmes (Becker, Goetz, Morger, \& Ranellucci, 2014), leur façon d'enseigner (Saunders, 2013), leur identité professionnelle (Hong, 2010) et même leur équilibre de vie en général (Schutz, 2014).

Or, à ce niveau, on observe d'importantes différences entre enseignants expérimentés et novices. Comme le montrent Pillen, Beijaard \& den Brok (2013) aux Pays-Bas, Shoffner (2011) aux Etats-Unis, Kyriacou \& Kunc (2007) en Angleterre, Ria \& Chaliès (2003) et Visioli \& Ria (2010) en France, le début de carrière en classe est marqué par l'immersion dans de véritables "bains de bulles" émotionnels qui peuvent conduire l'EN à ressentir une impuissance à agir face aux imprévus du métier, ce qui peut affecter sa construction identitaire et provoquer de la souffrance (Lantheaume \& Hélou, 2008 ; Méard \& Zimmermann, 2014). Ces situations émotionnellement marquantes contrastent avec le climat et les expériences vécues en centre de formation universitaire, où le curriculum de formation prévoit peu de dispositifs particuliers permettant d'atténuer ces bains de bulles répétés (Moussay, Malo \& Méard, 2012). Les EN ressentiraient des émotions, sinon spécifiques, du moins plus intenses que les enseignants chevronnés (Hargreaves, 1998 ; Ria, 2001) du fait des imprévus dans leur travail en classe (Gore \& Bowe, 2015) et de leur besoin régulièrement non satisfait sur le versant pratique de leur formation en alternance (Le Maistre \& Paré, 2010). Lors de leur entrée dans le métier, ils sont confrontés au choc d'une réalité qui réduit leur enthousiasme au fil des difficultés rencontrées (Kim \& Cho, 2014 ; De Mauro \& Jennings, 2016). Par exemple, la discipline dans la classe est un sujet de préoccupation, tout comme les échanges avec les parents, ce qui démontre à quel point 


\section{eJRIEPS 45 (Avril 2019)}

la volonté d'être pris au sérieux et respectés est centrale pour les EN (Avalos \& Aylwin, 2007).

Dès lors, comprendre et jouer avec ses propres émotions, augmenter ou réduire l'expression de ses émotions face aux élèves (Visioli et al., 2014), et être attentif à celles des élèves, constitueraient un axe central dans le développement professionnel des EN (Visioli \& Petiot, 2017). Mais, hormis dans les études relevant d'approches cliniques, peu d'informations sont délivrées par la littérature sur le processus d'évolution de ces savoirfaire relatifs à l'émotion chez les EN, même si certaines études visent à mesurer l'impact d'une inclusion de "compétences émotionnelles" dans les programmes de formation initiale (Burel, 2014).

Cette problématique paraît encore plus saillante en éducation physique et sportive (EPS). II semble en effet que cette discipline comporte des traits spécifiques relativement aux émotions des élèves qui interagissent de façon plus importante, qui peuvent osciller rapidement de l'enthousiasme à l'inhibition, et dont l'expression des émotions est peu contrôlable du fait de leur liberté de mouvement (Méard, 2013). Les supports sportifs et ludiques de l'EPS sont eux-mêmes porteurs d'une symbolique particulière qui réveille des réactions archaïques en rapport avec les différents espaces investis par les corps (Jeu, 1977). De leur côté, les enseignants d'EPS font face à davantage d'imprévus (relatifs à la sécurité, aux variations d'engagement des élèves, aux difficultés de supervision) que leurs collègues des autres disciplines (Zimmermann \& Méard, 2016). Ils sont eux-mêmes engagés corporellement (Ottet \& Méard, 2018). Plusieurs travaux récents relèvent d'approches situées (Visioli \& Ria, 2010), mais le plus souvent d'approches psychosociales, à propos du lien entre l'émotion et l'anxiété (Salavera, Antoñanzasb, Noéb \& Teruelb, 2014), du rapport entre l'enseignant novice (EN) et l'enseignant expérimenté (Aktop \& Beyazgül, 2014), des caractéristiques individuelles des enseignants (Colakoglua \& Yılmaz, 2014) ou encore de leur capacité à contrôler leur colère (Ayme, Ferrand \& Cogérino, 2011).

Parmi les études qualitatives portant sur les émotions en EPS auprès des EN, Ria et al. (2003) décrivent, à l'aide de la théorie sémiologique du cours d'expérience, auprès de huit enseignants débutants, des variations émotionnelles souvent en lien avec leur haut degré de dépendance à leurs préparations de leçons et leur souci de maintenir l'engagement des élèves. Ces deux préoccupations d'EN entraînent des émotions importantes dans les situations qui se situent au confluent de l'enseignement idéal planifié et de celui effectivement réalisé. Les EN font face à de nombreux imprévus (Bullough, 2009), et dans la mesure où leur capacité à anticiper les incertitudes est réduite, ces situations d'incertitude 


\section{eJRIEPS 45 (Avril 2019)}

non planifiées à l'avance provoquent chez eux des émotions intenses et souvent négatives (Derri, Papamitrou, Vernadakis, Koufou \& Zetou, 2014). Et c'est le premier objectif visé par l'étude que nous présentons ici: établir un panorama des différentes situations émotionnellement marquantes vécues par les EN en EPS.

Or, un des aspects saillants de tous ces travaux est que la charge émotionnelle y est présentée, de manière générale, comme un frein potentiel au développement, un obstacle, une réaction de survie présentant des entraves à une étape du développement marquée par « le manque de compétences et d'agentivité » (Johnson, Down, Le Cornu et al., 2014). A cette conception déficitaire (Malo, 2008) et assez « cartésienne » de l'impact de l'émotion sur le développement professionnel, celle-ci pourrait être considérée au contraire comme une clé du développement, selon une perspective inspirée de Spinoza. Dans cette optique, le pouvoir de développement est supposé être indissociable du pouvoir d'être affecté (Clot, 2017 ; Vygotski, 1931/1998). Mais la littérature internationale, là aussi, hormis les travaux relevant d'approches situées, apporte peu d'informations sur l'identification précise des effets de ces situations émotionnellement marquantes sur le développement professionnel des EN. Le deuxième objectif visé par notre étude consiste donc à mieux comprendre ce lien entre les situations émotionnellement marquantes vécues par les EN en EPS et leur développement.

Enfin, à propos de ce lien entre émotion et développement professionnel des EN, quelques travaux pointent la nécessité de mettre en place les conditions sociales d'échanges suscitant chez l'EN une réflexion, notamment à propos de ces situations particulières, en les partageant avec d'autres collègues dans un milieu soutenant et aidant (Park, Oliver, Johnson, Graham, \& Oppong, 2007). Les discussions avec les collègues plus expérimentés en salle des maîtres permettraient ainsi aux EN en EPS de s'ouvrir à propos de leurs satisfactions imprévues ou d'envisager d'autres possibilités face à des difficultés (Christensen, 2013 ; Cowie, 2011). En contexte francophone, ces éléments de la littérature internationale sont étayés par les nombreuses données de l'ergonomie et de la psychologie du travail qui pointent le rôle central du « collectif de travail » pour prévenir les problèmes de santé de l'opérateur (Clot, 2017). Il apparaît, en effet, que les enseignants isolés, c'està-dire ne pouvant partager leurs préoccupations (issues notamment de situations émotionnellement marquantes) avec autrui, seraient plus enclins à quitter la profession de manière prématurée (Hong, 2010). La proportion de partage de ces situations marquantes par les EN en EPS et leur développement professionnel représentera notre troisième objectif de recherche. 


\section{eJRIEPS 45 (Avril 2019)}

Suite à ces réflexions, nous avons donc réalisé une étude empirique cherchant à recenser et à établir une cartographie des situations émotionnellement marquantes vécues par les EN en EPS, les émotions ressenties et leur intensité. Autrement dit, le but était de faire un inventaire de ce qui affecte les EN dans cette discipline. De plus, nous avons tenté de mieux comprendre le lien éventuel entre ces situations et le développement professionnel des EN et nous avons cherché à savoir si les EN « partageaient » ces situations avec autrui.

\section{Méthode}

II s'est donc agi d'identifier les types de situations émotionnellement marquantes pour les EN en EPS, la nature et l'intensité des émotions ressenties dans ces situations, le partage de ces situations avec autrui et les effets déclarés de ces situations sur leur développement. Nous nous sommes inspirés de la démarche de Petiot, Visioli et Desbiens (2015), dans laquelle il était demandé à des enseignants de raconter deux événements marquants, positifs ou négatifs, survenus depuis le début de leur carrière. Cette étude contenait une taxonomie de huit inducteurs émotionnels. Nous avons emprunté ce questionnaire et l'avons adapté afin de pouvoir répondre à nos questions de recherche.

\subsection{Contexte et participants}

Cent trente-neuf enseignants d'EPS (92 hommes et 47 femmes, âgés de 25 à 36 ans) en première année de formation professionnelle initiale de la Haute Ecole Pédagogique (HEP) du Canton de Vaud (Lausanne, Suisse) ont donné leur accord pour participer à cette étude. Ils se destinaient à enseigner à des élèves du secondaire (élèves de 10 à 18 ans), tous suivaient un cursus constitué, d'une part d'enseignements, et d'autre part de stages dans des classes, sous la supervision de tuteurs. Chacun avait une histoire préprofessionnelle particulière, mais la majorité avait déjà effectué des remplacements d'enseignants d'EPS dans des établissements scolaires. Plus de la moitié d'entre eux avait déjà réalisé plus de 300 heures de remplacement, ce qui représente environ 15 semaines d'enseignement pour un professionnel en service. Comme ils avaient peu d'expérience professionnelle et n'étaient pas titularisés, nous les avons qualifiés d'enseignants novices (EN).

\subsection{Recueil de données}

Dans le questionnaire, l'EN devait rapporter par écrit deux situations émotionnellement marquantes, perçues comme positives ou négatives, vécues en tant qu'enseignant en situation de classe auprès des élèves (3 à 10 lignes chacune). Ensuite, il devait caractériser le type d'émotions ressenties (négativement ou positivement) sur le moment et leur degré d'intensité (sur une échelle de trois degrés, de "peu intense" à "très intense") afin d'avoir 


\section{eJRIEPS 45 (Avril 2019)}

des nuances dans les réponses des participants, sans pour autant utiliser les sept degrés habituels de l'échelle de Lickert. Comme ces récits avaient lieu a posteriori, les trois nuances nous sont apparues suffisantes pour documenter notre objet de recherche. Pour cela, nous nous sommes inspirés de la typologie des émotions primaires de Parott (2001) (surprise, joie, colère, tristesse et peur), proche de la TEI (Teacher Emotion Inventory) de Chen (2016) dont la fonction était moins de catégoriser a priori que de limiter le champ lexical des réponses et de pouvoir interpréter les données. Nous avons retiré la catégorie "amour" qui nous semblait inadéquate à propos de situations professionnelles, bien que des enjeux de séduction soient présents dans la relation enseignant-enseigné. Nous avons aussi laissé l'opportunité aux participants de mentionner d'autres émotions (rubrique “autres émotions"). La valence de chaque situation était déterminée en fonction de l'émotion ressentie, les émotions de colère, tristesse et peur colorant la situation négativement, alors que la joie la colorait de manière positive. La surprise, elle, donnait tantôt une valence positive, tantôt une valence négative, suivant les situations décrites, puisque l'émotion de surprise est qualifiée de mixte. Dans chaque situation pouvaient être mentionnées plusieurs émotions différentes. Les participants devaient également renseigner le chercheur sur le partage éventuel de chaque situation avec autrui, ce partage ayant pu être effectué auprès du milieu professionnel (collègues), auprès de la hiérarchie (directeur, adjoint de direction) ou encore auprès de la sphère privée (ami, conjoint). L'opportunité de noter d'autres interlocuteurs a également été laissée aux participants.

Enfin, l'EN devait relater les effets que chaque situation émotionnellement marquante avait eu sur leur développement, d'abord en inscrivant cet effet sur une échelle de sept degrés (de très négatif (-3) à très positif (3), en passant par aucun effet (0)), puis il devait préciser les éléments explicatifs de ces effets.

\subsection{Traitement des données}

Le but était de dresser un tableau des situations émotionnellement marquantes vécues par les EN en EPS. Face à la diversité des 278 situations recueillies, il semblait inapproprié de partir de catégories de situations préalables qui auraient pu être non pertinentes pour le contexte et le sujet. De là, nous avons cherché à « typifier » les situations selon la méthode de la "théorie ancrée" (Strauss \& Corbin, 1990) qui consiste à traiter de manière inductive des données et à les regrouper selon des traits de ressemblances. A propos du travail de classement de situations émotionnellement marquantes rapportées par des EN, il est paru inadéquat de définir des catégories (impliquant un périmètre étanche et des traits exclusifs). En effet, chaque situation pouvait être rapprochée à d'autres qui avaient des ressemblances 


\section{eJRIEPS 45 (Avril 2019)}

(par exemple, l'évocation par l'EN d'un élève en situation de handicap) mais de façon non exclusive (par exemple, sans parler des implicites, le fait que dans cette situation il y ait eu en filigrane un imprévu organisationnel). Pour cette raison, nous n'avons pas défini de catégories de situations, mais des «types». A cette fin, un codage approfondi en quatre étapes a été réalisé par deux chercheurs.

Etape 1 : transcription du récit

Nous avons d'abord retranscrit les situations évoquées par les EN, saisi numériquement les émotions et intensités, retranscrit et saisi le partage avec autrui et l'effet de chaque situation sur la suite de l'enseignement de l'EN en EPS.

Etape 2 : codage in vivo

Ce premier codage consistait à classer "par tas" les récits des sujets en gardant les formulations des acteurs eux-mêmes, conformément aux recommandations de Glaser \& Strauss (1978).

Etape 3 : codage axial

Ce second codage visait à regrouper et à étiqueter (par les chercheurs) les situations rapportées selon des «types » (Strauss \& Corbin, 1990).

Etape 4 : codage sélectif

A cette étape du traitement des données, le but était de construire un type de situation central qui rende significatif tous les types de situations émotionnellement marquantes préalablement mises à jour. Elle constitue l'étape finale des résultats qui peuvent être confrontés et discutés avec ceux de la littérature (voir encadrés 1 et 2).

\section{EXEMPLE 1 (situation 125b)}

Etape 1 (Transcription de la situation) :

Avec mes 10VG, nous avions fait un test d'endurance, type navette avant les vacances, et les résultats étaient très désolants. Seulement quatre personnes avaient atteint le barème "réussi". Du coup, je les avais prévenus que novembre serait le mois de la condition physique et de l'endurance, et à travers des jeux et séances en musique, nous avons entraîné cette endurance. Au début c'était très difficile, il fallait que je les menace pour qu'ils se mettent à courir, ils ne voulaient pas entrer dans cette logique d'entraînement, puis petit à petit ils ont pris goût et finalement lorsque nous avons refait le test, tout le monde a réussi à atteindre son palier.

Etape 2 (codage in vivo) : Les élèves se sont améliorés de manière significative lors de tests de condition physique 


\section{eJRIEPS 45 (Avril 2019)}

Etape 3 (codage axial) : réussites d'un ou plusieurs élèves

Etape 4 (codage sélectif) : apprentissages effectifs des élèves

\section{EXEMPLE 2 (situation 112b)}

Etape 1 (Transcription de la situation rapportée) :

Pendant mon stage, la gestion d'une classe de 10VP s'avère être parfois compliquée. Ma Prafo (ndlr. : tutrice) n'était pas présente, parce qu'elle avait fait exprès d'arriver 10/15 minutes après le début du cours pour voir comment ça se passe sans sa présence. Pendant ce cours (leçon pendant laquelle j'ai fait des jeux), deux élèves avaient un comportement incivilisé. Les deux élèves ont clairement influencé tous les autres garçons. La leçon ne s'est pas bien passée parce que j'ai dû me concentrer sur la gestion de classe au lieu de l'apprentissage.

Etape 2 (codage in vivo) : Comportement incivilisé d'élèves qui influencent les autres garçons de la classe. Leçon qui ne se déroule pas bien

Etape 3 (codage axial) : transgression volontaire en opposition collective Etape 4 (codage sélectif) : transgressions de règles par un ou plusieurs élèves

Ces différentes étapes du processus de codage, réalisées successivement par deux chercheurs, ont été regroupées dans un tableau à six colonnes (voir tableau 1).

Tableau I : Exemple des étapes successives de la procédure de codage

\begin{tabular}{|c|c|c|c|c|}
\hline \multicolumn{2}{|c|}{ EXEMPLE 3 : (situation 135a) } & \multicolumn{1}{|c|}{$\begin{array}{l}\text { Codage } \\
\text { in vivo }\end{array}$} & Codage axial & Codage sélectif \\
\hline$N^{\circ}$ & Transcription & Accident d'élève & $\begin{array}{l}\text { Risques relatifs à } \\
\text { l'intégrité physique } \\
\text { des élèves }\end{array}$ \\
$\begin{array}{l}\text { 135a } \\
\text { s'est ouverte le crâne sur un } \\
\text { chariot que j'ai laissé traîner } \\
\text { par inadvertance et n'a pas } \\
\text { voulu arrêter de jouer pour } \\
\text { défier mon autorité et a } \\
\text { arrosé la salle de sang. J'ai } \\
\text { dû l'arrêter manu militari et } \\
\text { le forcer à être accompagné } \\
\text { à l'infirmerie. }\end{array}$ & $\begin{array}{l}\text { Une élève } \\
\text { s'ouvre le crane } \\
\text { sur un chariot } \\
\text { laissé dans la } \\
\text { salle }\end{array}$ & & \\
\hline
\end{tabular}

Concernant les autres données, il s'est agi d'identifier des liens entre les types de situations, les émotions et leur intensité. Les fréquences d'apparition de chaque émotion (peur, colère, 


\section{eJRIEPS 45 (Avril 2019)}

tristesse, surprise, joie) ainsi que leurs intensités respectives dans les différents types de situations ont donc été calculées et rapportées dans un tableau récapitulatif faisant apparaître les occurrences et les pourcentages.

Les «partages » de ces situations avec autrui ont ensuite été comptabilisés au total puis mis en rapport avec les différents types de situations et les différents interlocuteurs (collègues, tuteurs, conjoints, amis, hiérarchie) selon leurs occurrences.

Enfin, les effets des situations marquantes déclarés par les EN sur leur développement ont été répertoriées puis classées selon leur sept degrés (de très négatif (-3) à très positif (3), en passant par aucun effet (0)). Ces données ont été traitées à l'aide de statistiques descriptives, fréquences et écarts-types.

\section{Résultats}

Dans un premier temps (3.1), les différents types de situations émotionnellement marquantes vécues par les EN en EPS seront présentés sous forme d'un tableau dont les éléments saillants seront commentés. La partie suivante (3.2) sera consacrée aux rapports entre les types de situations vécues, les émotions et leurs intensités. Dans la troisième partie (3.3) seront exposés les résultats relatifs aux effets déclarés des situations vécues et au partage avec autrui. Enfin, la quatrième partie des résultats (3.4) présentera le cas particulier des 18 situations à l'origine de « revirements émotionnels » rapides car nous considérons qu'elles sont éclairantes d'un point de vue qualitatif.

3.1 Le type des situations émotionnellement marquantes vécues par les EN A la suite du codage sélectif, 12 types de situations émotionnelles vécues par les EN ont été identifiés. Le tableau II présente ces 12 types ainsi que la valence (émotion plutôt positive ou négative) attribuée par les EN, leur nombre et leur pourcentage respectifs. On observe que six types sont à valence négative, cinq à valence positive. Un des types de situation est celui des actions d'élèves particuliers, qui suscite des émotions positives dans 21 situations sur 28, négatives dans sept situations sur 28 .

Tableau II : Les types de situations émotionnellement marquantes vécues par l'EN (codage sélectif)

\begin{tabular}{|c|l|c|c|}
\hline Situation & \multicolumn{1}{|c|}{ Types de situations } & Nombre & Total \\
\hline négative & Transgressions de règles par les élèves & 78 & $28.1 \%$ \\
\hline négative & Risques relatifs à l'intégrité physique des élèves & 58 & $20.9 \%$ \\
\hline
\end{tabular}


eJRIEPS 45 (Avril 2019)

\begin{tabular}{|c|l|c|c|}
\hline négative & Actions non motivées d'élèves (par le travail scolaire) & 10 & $3.6 \%$ \\
\hline négative & Imprévus organisationnels ou matériels & 9 & $3.2 \%$ \\
\hline négative & Manques de complicité entre l'EN et les élèves & 8 & $2.9 \%$ \\
\hline négative & Décalages avec l'équipe des professionnels & 5 & $1.8 \%$ \\
\hline négative & \multirow{2}{*}{ Actions d'élèves particuliers } & 21 & $7.6 \%$ \\
\hline positive & & 7 & $2.4 \%$ \\
\hline positive & Actions motivées d'élèves (par le travail scolaire) & 42 & $15.1 \%$ \\
\hline positive & Apprentissages effectifs des élèves & 25 & $9 \%$ \\
\hline positive & Complicités entre l'EN et les élèves & 10 & $3.6 \%$ \\
\hline positive & Suivi de ce qui était prévu & 3 & $1.1 \%$ \\
\hline positive & Inclusions dans l'équipe des professionnels & 2 & $0.7 \%$ \\
\hline Totaux & & 278 & 100 \\
\hline
\end{tabular}

Les EN en EPS rapportent davantage de situations associées à des émotions négatives (181/278, soit $65.1 \%$ des réponses) que positives (79/278, soit $28.4 \%$ des réponses). Concernant les situations émotionnellement marquantes identifiées à tonalité négative par les EN, plusieurs types récurrents émergent : les transgressions de règles de la part d'un ou plusieurs élèves (78/278, soit $28.1 \%$ des réponses) représentent le type de situation le plus fréquent, par exemple, quand un groupe d'élèves perturbe la classe, ou quand un élève refuse explicitement de pratiquer, discute et rit, comme dans la situation 86a, lorsque « Trois élèves perturbent le groupe en faisant n'importe quoi (se pendre au but, shooter un ballon n'importe où etc.). J'ai dû les sortir du cours les dix dernières minutes et discuter de cela avec eux. "

Les risques relatifs à l'intégrité physique des élèves (58/278, soit 20.9\% des réponses) représentent également une part importante des situations émotionnelles rapportées par les EN, comme l'illustre la situation 6a : "Alors que les élèves semblaient en sécurité, un accident est arrivé. J'insiste sur les dangers et la sécurité depuis ce jour-là dans toutes les activités. Quand les élèves sont trop engagés, je leur dis de faire attention aux règles de sécurité. »

D'autres situations dans lesquelles se manifestent des actions d'élèves non motivées par le travail scolaire, moins fréquentes, sont également ressenties négativement par les EN (10/278 - 3.6\%), comme dans la situation 35b : «Durant un jeu de basketball, un élève s'assoit sur le banc. Je pense qu'il se sent mal mais il me dit : "Je suis fatigué de jouer. 


\section{eJRIEPS 45 (Avril 2019)}

J'arrête, je suis fatigué". J'ai discuté avec lui deux minutes, et j'ai essayé de l'encourager, de le motiver. Finalement il s'est levé. Je ressentais de la colère et de la crainte car j'ai eu peur qu'il reste là et ne bouge pas. ”

Les EN sont fortement surpris lors de ce type de situations négatives (148/278, soit 53.2\% des situations), quand un accident survient, quand les élèves refusent de participer, transgressent les règles ou encore dans des situations qu'ils jugent imprévisibles. Cette surprise est exprimée, par exemple, dans la situation 47a : « Lundi matin, on m'a informé que je ne pouvais pas utiliser la salle de sport parce qu'elles n'étaient pas disponibles (rangements d'une manifestation ayant eu lieu le week-end). Cinq minutes plus tard, nouvelle information : ma collègue était malade et j'étais supposé enseigner à 40 élèves en extérieur alors qu'il pleuvait et qu'il faisait froid. ”

Quant aux situations émotionnelles ressenties positivement par les EN, elles sont pour beaucoup d'entre elles associées à des actions motivées d'élèves (42/278, soit 15.1\% des réponses) et aux apprentissages effectifs d'élèves (25/278, soit $9 \%$ des réponses). A ce sujet, nous pouvons remarquer que les actions d'élèves particuliers, notamment en raison de handicaps, d'inaptitudes non anticipées ou invisibles, sont regroupées dans le même type de situations, ressenties soit positivement, soit négativement. Parfois, c'est la joie (90/278, soit 32,4\% des réponses) qui semble renforcer chez l'enseignant son sentiment d'avoir infléchi la situation (seules 5 situations vécues ayant procuré de la joie semblent n'avoir aucun effet sur le développement professionnel), même si la plupart du temps, c'est la tristesse (13/28 dans le type de situation absences d'apprentissage d'élèves particuliers, soit 46,24\% des réponses pour ce type) parce que "le mal a été fait" (l'élève non repéré a chuté ou a échoué). Ce type, libellé « actions de cas d'élèves particuliers », représente chez les EN en EPS un vécu émotionnellement marquant à part entière que nous ne saurions associer, dans un souci de transparence, à d'autres types. Le lien entre toutes les situations émotionnellement marquantes issues de ce type est caractérisé justement par la dimension particulière, « hors norme » d'un ou plusieurs élèves.

3.2 La nature et l'intensité des émotions ressenties dans les situations

Cette étude ne permet pas d'associer systématiquement tel type de situations et tel type d'émotions, même si, comme le montre le tableau III, nous constatons que la surprise est l'émotion qui apparaît le plus fréquemment, à moyenne ou haute intensité, alors que la tristesse est plutôt ressentie à faible ou moyenne intensité, au contraire de la joie qui est, elle, ressentie de manière très intense. L'intensité de la peur est répartie de façon équilibrée 


\section{eJRIEPS 45 (Avril 2019)}

entre les 3 degrés, alors que la colère est le plus souvent ressentie avec une intensité moyenne.

Tableau III : La fréquence d'apparition de chaque émotion dans chaque type de situation

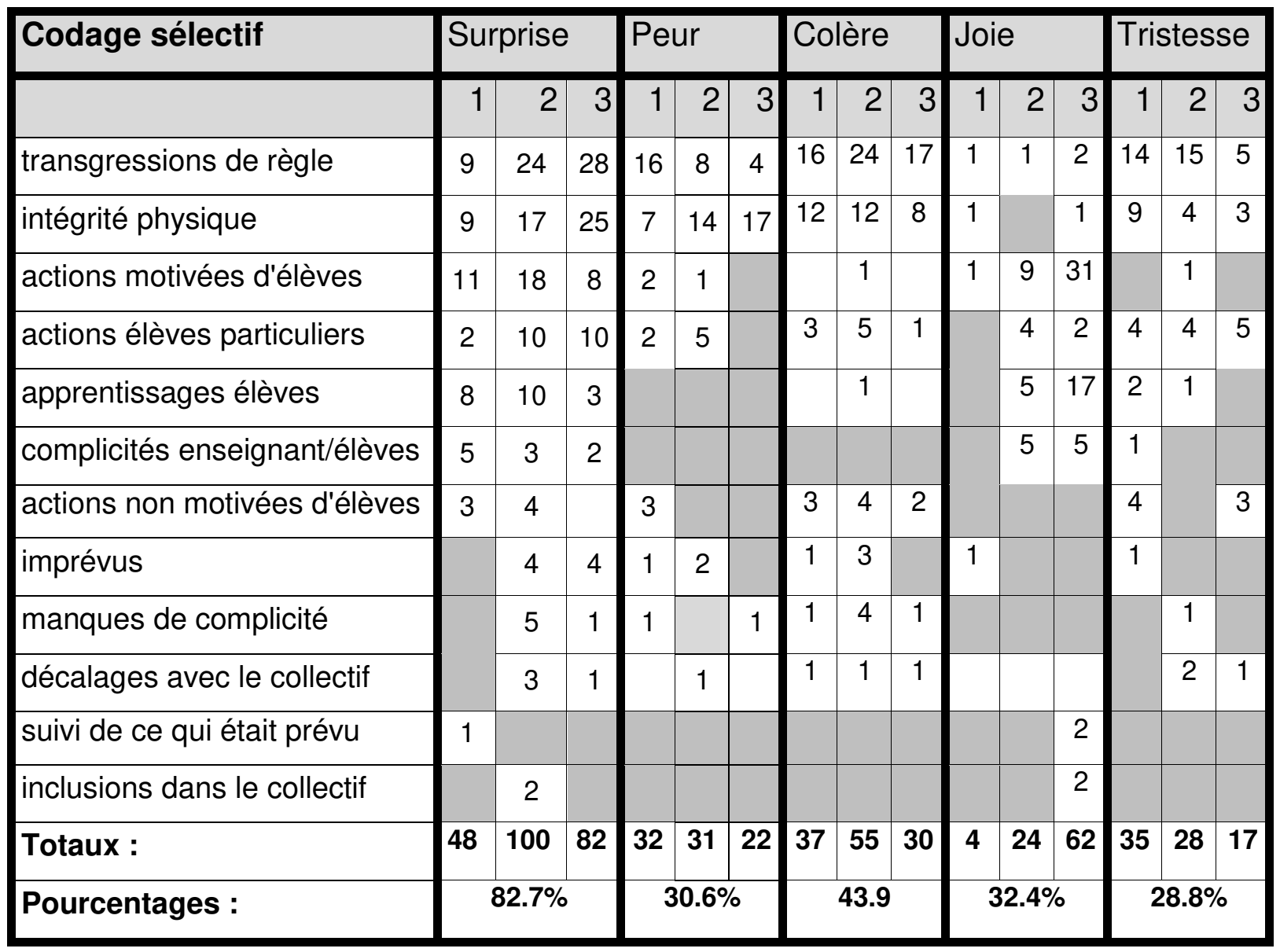

Ainsi, la surprise peut être positive ou négative. Elle est présente dans plus de $82 \%$ des situations évoquées et pas seulement à propos de celles relevant des types imprévus ou risques relatifs à l'intégrité physique.

On observe également que l'intensité de la surprise est importante lors « d'actions d'élèves particuliers ». Qu'il s'agisse d'une élève en situation porteuse d'un handicap non perçu au départ par l'enseignant, d'un élève dépressif qui pleure soudainement, ou encore d'un élève à propos de qui l'EN apprend qu'il est atteint d'une maladie incurable et mourra dans un avenir proche, ces situations marquent émotionnellement les EN en EPS. Comme dans la situation 94a, lorsqu'un élève confie à l'EN une partie de son histoire familiale, « très lourde dans laquelle il a d'u s'interposer face à son père qui battait sa mère. Il a même été jusqu'à le dénoncer et son papa se trouve maintenant en prison. Il venait de l'avoir au téléphone, mais il a refusé de lui parler ». A l'inverse, ce type de situation, lorsqu'il débouche sur ce 


\section{eJRIEPS 45 (Avril 2019)}

que l'EN perçoit comme une réussite (un regain d'engagement, un apprentissage effectif de l'élève), provoque chez l'EN des pics émotionnels positifs.

Sur le versant négatif, la colère est présente (43.9\%), ainsi que la peur (30.4\%) et la tristesse (28.8\%). Au regard de l'intensité de l'émotion, il est difficile de tirer des conclusions définitives: la tristesse est ressentie avec peu d'intensité, la colère avec une intensité moyenne, la surprise aussi. Ces émotions, ainsi que leur intensité semblent souvent liées dans les descriptions de situations au sentiment de l'EN qu'il « ne peut rien faire ", à un sentiment d'impuissance.

La joie (32.4\%) apparaît lorsque les élèves sont engagés, quand ils apprennent et se développent. Quand les enseignants la ressentent, c'est la plupart du temps avec une intensité forte, surtout quand la situation débute difficilement : "Cette leçon a été difficile à gérer à cause de niveaux différents d'élèves et de situations problématiques. Mais certains élèves se sont engagés et ont finalement bien réalisé des habiletés qu'ils pensaient impossibles au début du cycle. J'ai ressenti une joie forte au cours de cette leçon » (situation 24b).

3.3 Les effets des situations sur le développement et le partage avec autrui Relativement aux effets de ces situations émotionnellement marquantes sur le développement professionnel, les EN déclarent dans plus de $83 \%$ des cas (233/278) que ces situations émotionnelles ont un impact positif (tableau IV). Ce résultat est relativement surprenant au vu des éléments de la littérature.

Tableau IV: L'effet déclaré des situations émotionnellement marquantes sur le développement professionnel 


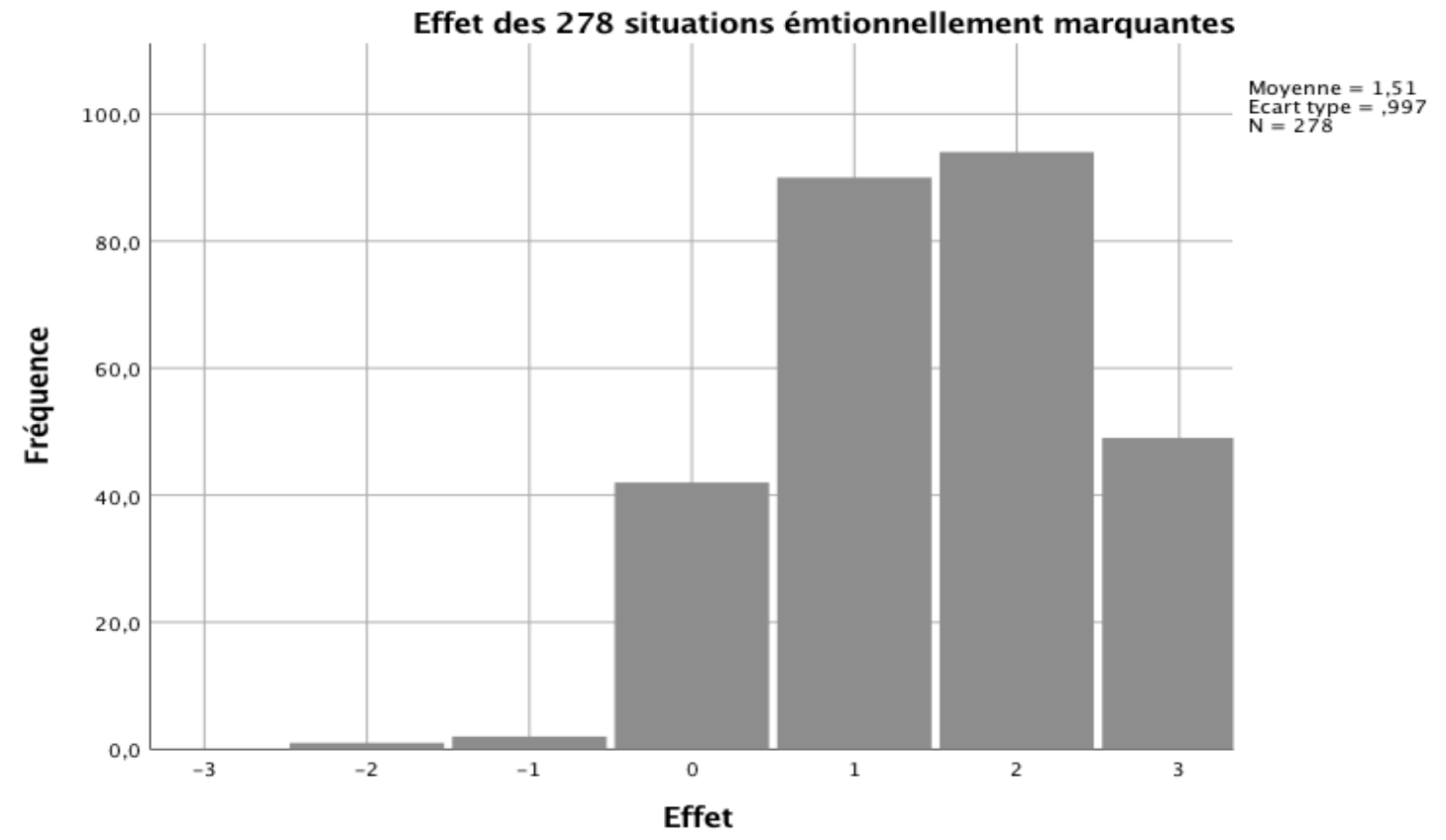

Même si ces données sont déclaratives, ce résultat indique qu'un processus de changement s'opère la plupart du temps chez les EN dans leur manière d'enseigner à la suite de ces situations émotionnellement marquantes (tableau VI). Ressentie sur le moment comme négative, une situation peut déclencher une réflexion et inciter l'EN à opérer des réajustements pour que le problème ne ressurgisse pas : "Face à ce constat d'échec, j'ai dû rapidement changer mon enseignement et mes choix pour trouver des solutions " (situation 2a). Une EN mentionne que "chaque erreur est une source d'apprentissage, on doit réfléchir à sa pratique et en tirer les conclusions pour ajuster et apporter des changements la fois suivante " (situation 52b). Le tableau suivant nous renseigne effectivement sur l'effet positif de la plupart des situations vécues, en fonction de leur valence positive ou négative (tableau $\mathrm{V}$ ).

Tableau V : L'effet des situations selon leurs valences respectives

\begin{tabular}{|l|r|r|r|r|}
\hline $\begin{array}{c}\text { Situation émotionnellement } \\
\text { marquante }\end{array}$ & Effet négatif & Pas d'effet & Effet positif & Total \\
\hline négative & 3 & 37 & 141 & 181 \\
\hline négative puis positive & 0 & 0 & 18 & 18 \\
\hline positive & 0 & 5 & 74 & 79 \\
\hline Total & 3 & 42 & 233 & 278 \\
\hline
\end{tabular}




\section{eJRIEPS 45 (Avril 2019)}

Les situations vécues négativement ou très négativement ne semblent donc pas représenter un obstacle au développement professionnel, au contraire, puisque seule une très faible quantité d'émotions vécues conduit à un effet néfaste sur le développement professionnel (3/278, soit $1.1 \%$ des émotions négatives).

Les EN déclarent aussi dans une moindre mesure que certaines situations émotionnellement marquantes ont un effet neutre sur leur développement (42/278, soit $15,1 \%$ des réponses), mettant en avant le caractère singulier de la situation, comme pour cet EN (situation 53a) qui reçoit le même jour une visite certificative de son tuteur et d'un didacticien du centre de formation : «Ce fut un événement ponctuel. J'ai appris certaines choses importantes, mais le principal était de se relever et de penser à la suite. Je voulais montrer que j'étais capable de nettement mieux ». Ou encore cet EN (situation 42a) qui est face à « un élève arrivant en cours avec une arme le lendemain des attentats de Paris. L'arme était factice, mais en métal, de taille et poids réels ». Ces deux situations, bien qu'émotionnellement marquantes pour l'EN (colère d'intensité $3 / 3$ pour la première et surprise d'intensité 3/3, colère d'intensité $2 / 3$ et peur d'intensité 2/3 pour la seconde) n'ont, d'après le participant, pas eu d'effet sur son développement car cela reste un cas marquant, mais isolé (situation 42a), même si le premier extrait laisse à penser que l'EN a « appris certaines choses » qui pourraient néanmoins représenter un vecteur de développement. Seules trois situations émotionnellement marquantes ont un effet négatif sur les EN (3/278, soit $1.1 \%$ des réponses). L'EN y ressent de la colère, de la tristesse (1a et 136a) et du dégoût, soit parce que son tuteur lui demande de réaliser à l'improviste auprès des élèves une activité qui dégénère, soit parce qu'il a l'impression que son statut d'enseignant d'EPS est « dévalorisé vis-à-vis des autres enseignants", soit à cause d'une réaction d'élève (« c'est nul votre exercice ») qui le fait « culpabiliser».

Les effets sur le développement professionnel peuvent aussi être observés en regard des différents types de situation issus du codage sélectif (tableau VI).

Tableau VI : L'effet des différents types de situations émotionnellement marquantes

\begin{tabular}{|l|r|r|r|r|}
\hline \multicolumn{1}{|c|}{ Codage sélectif } & \multicolumn{1}{c|}{$\begin{array}{c}\text { Effet } \\
\text { négatif }\end{array}$} & \multicolumn{1}{c|}{$\begin{array}{c}\text { Pas } \\
\text { d'effet }\end{array}$} & $\begin{array}{c}\text { Effet } \\
\text { positif }\end{array}$ & \multicolumn{1}{|c|}{ Totaux } \\
\hline transgressions de règle & 1 & 15 & 62 & $\mathbf{7 8}$ \\
\hline intégrité physique & 0 & 9 & 49 & $\mathbf{5 8}$ \\
\hline actions non motivées d'élèves & 1 & 1 & 8 & $\mathbf{1 0}$ \\
\hline imprévus & 0 & 0 & 9 & $\mathbf{9}$ \\
\hline
\end{tabular}


eJRIEPS 45 (Avril 2019)

\begin{tabular}{|l|r|r|r|r|}
\hline manque de complicité & 0 & 5 & 3 & $\mathbf{8}$ \\
\hline décalages avec le collectif & 1 & 2 & 2 & 5 \\
\hline actions d'élèves particuliers & 0 & 6 & 22 & $\mathbf{2 8}$ \\
\hline actions motivées d'élèves & 0 & 0 & 42 & $\mathbf{4 2}$ \\
\hline apprentissages effectifs élèves & 0 & 3 & 22 & $\mathbf{2 5}$ \\
\hline complicités enseignant/élève & 0 & 1 & 9 & $\mathbf{1 0}$ \\
\hline suivi de ce qui était prévu & 0 & 0 & 3 & $\mathbf{3}$ \\
\hline inclusions dans le collectif & 0 & 0 & 2 & $\mathbf{2}$ \\
\hline totaux & $\mathbf{3}$ & $\mathbf{4 2}$ & $\mathbf{2 3 3}$ & $\mathbf{2 7 8}$ \\
\hline
\end{tabular}

En comparant ce tableau VI et le tableau II, on observe que les proportions et nombres respectifs des effets des situations correspondent à ceux des différents types de situations (autrement dit, les situations vécues comme négatives émotionnellement lors de transgressions de règles ou de problèmes liés à l'intégrité physique étant les plus nombreuses, ce sont elles qui conduisent à avoir le plus d'effet). On observe que ces effets déclarés semblent indépendants de la valence émotionnelle positive ou négative des situations concernées.

Les données mettent aussi en évidence que les situations émotionnellement marquantes sont partagées avec des interlocuteurs à une forte proportion (93.5\%, soit 260/278). Plus de la moitié le sont avec le tuteur (51.8\%) ou avec des collègues (51.8\%) mais un tiers sont partagées avec d'autres interlocuteurs qui ne sont pas du métier (conjoint, amis) (tableau VII). En revanche, la hiérarchie n'est impliquée dans ces échanges que dans $11.6 \%$ des situations. 


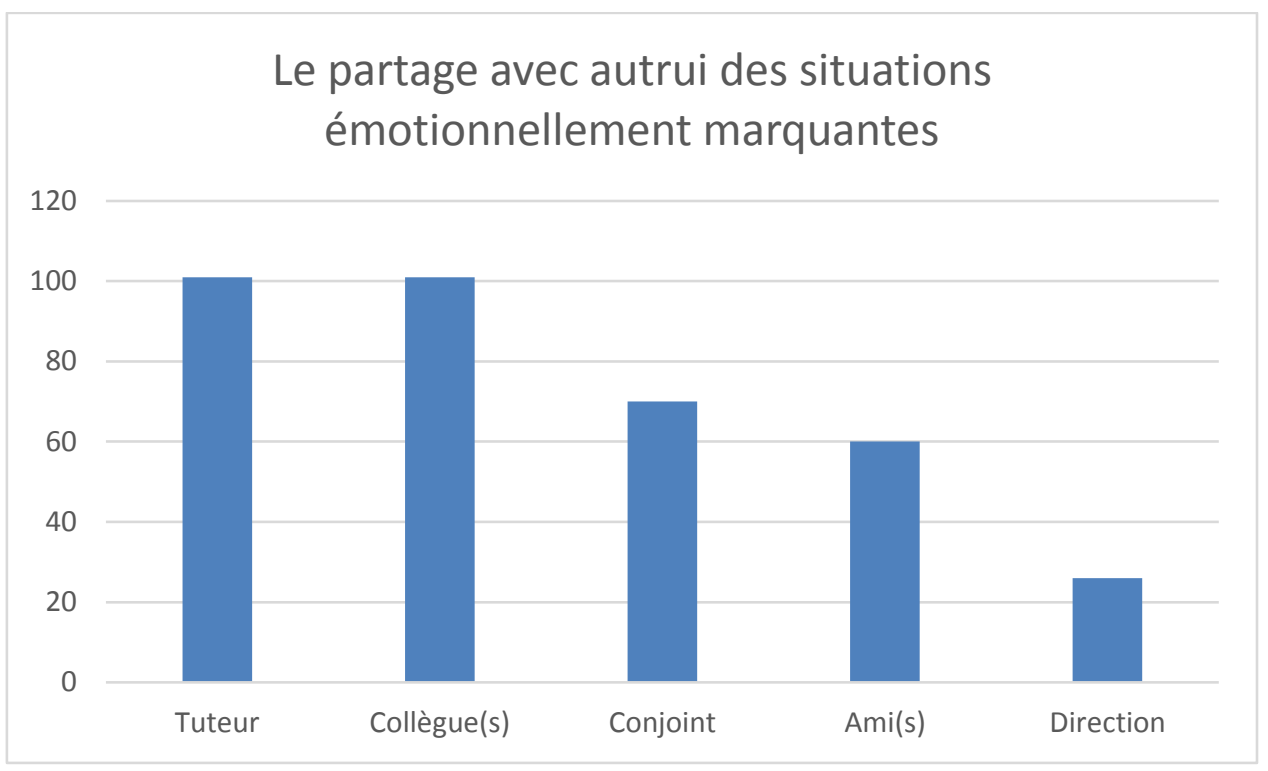

Figure 1 : Le partage avec autrui des situations émotionnellement marquantes vécues

Le croisement des situations partagées et des effets déclarés des situations émotionnellement marquantes sur le développement professionnel ne permet pas d'établir de lien entre les deux. Plus précisément, les situations dont les EN déclarent qu'elles n'ont pas d'effet ou un effet négatif ne sont pas moins partagées avec autrui que celles qui sont présentées comme ayant un effet positif.

\subsection{Les situations de revirements émotionnels}

Un certain nombre de situations sont particulières, dans le sens où elles sont ressenties au départ négativement, puis l'EN rapporte une issue positive à court ou long terme (16/278) (aucune situation n'est en revanche présentée d'abord positive, puis négative). Ces situations dont l'EN rapporte l'issue heureuse, tantôt avec effet immédiat grâce à une action sur le moment par l'EN (6/278), tantôt avec un effet différé grâce à des actions successives, sont illustrées notamment par cet EN (situation 43b) : «Elève peu sportive, régulièrement blessée (...). Je lui transmets le document "pratiquer une activité physique malgré une dispense ». Elle ne s'y attendait pas et a dû se plier à cette contrainte. Depuis, elle continue à effectuer ce programme et à se gérer (je peux l'observer). Elle prend du plaisir et est plus active de manière générale ". Dans cette situation, l'EN reprend la main sur une situation initialement délicate qui a provoqué chez lui colère $(1 / 3)$ et déception $(1 / 3)$ et parvient à surmonter cette difficulté liée à une inaptitude en mettant en œuvre un dispositif. L'effet est ensuite ressenti de manière positive, puisque cet EN dit « je prends toujours avec moi le dossier, même avec les autres classes. Je suis content de voir que l'élève a finalement adhéré à l'activité. Je voyais ceci comme un travail supplémentaire, une sanction. Il s'est 


\section{eJRIEPS 45 (Avril 2019)}

finalement avéré très pédagogique ». Alors que dans le cas présenté en amont, le processus mis en place a un effet différé, dans les extraits suivants, l'EN met en place le processus au cours même de la leçon, comme dans cette « classe de garçons réputée très compliquée à gérer. Badminton, beaucoup de réticence au début, quelques tentatives de "sabotage" de la leçon. Discussion avec le leader qui comprend son rôle, s'engage davantage et contribue à la réussite de la leçon » (situation 31a). L'effet relaté ici est d'intensité moyenne et positif (2/3) puisque l'EN met en évidence que pour la première fois, il a «réalisé la valeur et le potentiel d'un élève dans la réussite de ma mission d'enseignant. ”

Parfois, cette évolution d'émotion, du négatif au positif, est provoquée par les collègues de I'EN (situation 79a) : «Les enseignants d'EPS étaient encore en train d'installer le matériel (plusieurs postes) quand les étudiants sont arrivés en salle. Aucune prise en charge durant quelques minutes (4) de la part des enseignants. Les élèves sont restés calmes et discutaient. Cette non prise en charge m'a étonnée. C'était un moment de surprise au début négatif car de la part des enseignants c'est un manque d'anticipation et d'organisation de mon point de vue. Mais par la suite le fait que les étudiants n'ont pas profité de cette nonprise en charge pour faire les fous m'a surpris cette fois-ci positivement. " L'effet est de faible intensité, mais positif pour l'EN qui indique, que cette situation l'a fait « réfléchir et voir que lorsque l'on ne prend pas en charge les élèves, c'est possible que ça se passe bien mais je reste convaincue que notre salle doit être prête quand les élèves arrivent ou qu'on leur demande de l'aide pour finir de mettre le matériel. Dans tous les cas, je suis plus à l'aise lorsque les élèves sont pris en charge (il y a moins d'ennui et donc moins de conflits). ”

Ces différentes situations laissent penser que l'EN témoigne d'une forme de « développement en condensé », ce qui confirme finalement les premiers résultats présentés plus haut dans le tableau VI où, de façon contre-intuitive, on observe que la quasitotalité des situations, y compris celles qui sont ressenties très négativement d'un point de vue émotionnel, conduisent à des effets déclarés positifs sur le développement des EN. Les revirements émotionnels rapportés constituent une sorte d'exemple en raccourci d'adaptation, voire de « riposte », suite à une situation négative au départ.

\section{Discussion}

Ces résultats apportent des réponses d'inégal intérêt aux objectifs de recherche visés au départ : identifier les types de situations émotionnellement marquantes pour les EN en EPS, 


\section{eJRIEPS 45 (Avril 2019)}

la nature et l'intensité des émotions ressenties dans ces situations, le partage de ces situations avec autrui et les effets déclarés de ces situations sur leur développement.

Premièrement, ils démontrent une grande variété de situations susceptibles de susciter des états émotionnels chez les EN. La majorité de ces situations, en classe, est plutôt associée à des émotions négatives (les deux tiers environ). Ces résultats confirment ceux de Visioli et Desbiens (2015) dans lesquels il apparaît que l'inducteur émotionnel négatif principal se rapporte aux comportements non appropriés d'un ou plusieurs élèves en classe. Ils se distinguent en revanche de ceux de Chen (2016) selon laquelle les émotions agréables découleraient plutôt des situations de classes et des interactions entre collègues, alors que les moins agréables seraient plutôt en lien avec les prescriptions, les réformes et un déséquilibre dans la vie des enseignants. Dans la mesure où le contexte scolaire vaudois, dans lequel a été réalisée l'étude, ne semble pas particulièrement violent, transgressif, ni tendu socialement, la dissonance entre les résultats de l'étude de Chen et la nôtre pourrait être liée à la discipline EPS, particulièrement dans des situations où l'intégrité corporelle des élèves est mise à mal, où les imprévus, l'absence d'engagement et les transgressions de règles sont plus importants que dans d'autres disciplines (Méard, 2013). Par ailleurs, on observe, comme Ria et al. (2003), que la surprise est fortement présente dans le tableau émotionnel des EN, soit dans plus de quatre situations sur cinq. Les inattendus au cours de la leçon semblent constituer le substrat du "choc de la réalité" mis en évidence par de nombreux auteurs (Bullough, 2009 ; Kim \& Cho, 2014 ; De Mauro \& Jennings, 2016).

Deuxièmement, notre étude montre, de façon inattendue, que les situations émotionnellement marquantes vécues par les EN en EPS, y compris celles qui ont suscité une émotion négative forte, ont la plupart du temps, un effet positif déclaré sur le développement professionnel. Même si la méthode du questionnaire qualitatif ne recueille que des effets "déclarés ", le fait que la démarche repose sur l'évocation de situations vécues parfois plusieurs semaines ou mois plus tôt permet à chaque acteur de revisiter la situation et de rapporter le processus qui lui a succédé. Ce résultat contre-intuitif contribue, selon nous, à conforter la thèse d'une « émotion nécessaire » dans le processus de développement des EN, à la suite des options spinozistes initiées par Vygotski (1931-1998). Loin d'une optique cartésienne et cognitiviste de l'apprentissage du métier dans laquelle l'émotion représenterait un frein, elle se présente, ici, comme une condition du développement humain (Johnson et al., 2014). Pour paraphraser Clot (2017), le développement du pouvoir d'agir semble en quelque sorte indissociable du pouvoir d'être affecté. 


\section{eJRIEPS 45 (Avril 2019)}

Troisièmement, les résultats de notre étude ne font pas apparaître de liens entre les types de situations et les types d'émotions. Comme indiqué dans la partie 3.2 et le tableau III, il est impossible de tirer des conclusions à ce niveau, ni d'établir des associations entre les types de situations et les types d'émotions d'une part, ni l'intensité de ces émotions d'autre part. Ce constat, apparemment décevant, nous incite à poser que, finalement, le ressenti émotionnel est irréductiblement personnel (Damasio, 1994). Autrement dit, quel que soit le type de configuration dans lequel l'acteur se trouve placé, la subjectivité manifestée par une forme et une intensité émotionnelle donnée, est un substrat fondamentalement individuel qui échappe à la comptabilité d'un collectif (Ria et al., 2003). De plus, si l'on met en lien cette absence de corrélation entre situations, émotions et intensités d'un côté, et la proportion importante d'effets déclarés par les EN concernant leur développement professionnel de l'autre côté, on peut avancer que "les situations émotionnellement marquantes, quelle que soit leur spécificité et indépendamment des émotions qu'elles suscitent, conduisent la plupart du temps à favoriser pour les EN en EPS leur développement professionnel ».

Quatrièmement, notre étude met en évidence que les situations émotionnellement marquantes vécues en EPS par les EN sont massivement partagées avec le tuteur et avec les collègues, dans une moindre mesure avec la hiérarchie, des amis ou le conjoint. A la suite de certains auteurs qui mettent en évidence que l'expérience accumulée diminue le besoin de partage avec autrui, nos données auprès des EN pointent la proportion importante des expériences émotionnelles partagées avec autrui. Ces résultats interrogent les conclusions de Jokikokko et al. (2017), pour qui, dans les cas où l'émotion est très intense, les EN adopteraient la stratégie du silence. Alors que la recherche de Lassila et Uitto (2016) évoque plutôt des tensions entre enseignants expérimentés et EN lors de situations délicates vécues par les seconds, les 278 situations émotionnelles de notre étude témoignent à l'inverse que le partage avec autrui concerne plutôt les " gens de métier ", ceci permettant aux EN de faire face à ce vécu émotionnel (Moussay et al., 2012). De là, bien qu'il ne soit pas possible, là non plus, d'établir des liens, type de situations par type de situations, entre le partage avec autrui et les effets de ces situations déclarés par les EN sur leur développement professionnel, on peut être tenté néanmoins de les mettre en rapport globalement. En effet, on observe en même temps que la grande majorité des situations vécues (260/278) est discutée avec des interlocuteurs et qu'une proportion également importante de ces situations favorisent le développement professionnel, selon les EN (233/278). La juxtaposition de ces deux résultats globaux va selon nous dans le sens 


\section{eJRIEPS 45 (Avril 2019)}

des conclusions de travaux qui ont souligné l'importance pour les EN des échanges entre collègues (Christensen, 2013 ; Cowie, 2011) et d'un contexte professionnel soutenant (Park et al., 2007).

Enfin, cette étude fait apparaître quelques grands axes qui permettent à notre avis d'approfondir la compréhension des situations émotionnellement marquantes vécues par les EN en EPS. La première grande tendance tient selon nous dans le fait que la majorité des situations rapportées par les EN (181/278, soit 65.1\% des réponses) conduisent à terme à un sentiment de ne pas pouvoir agir, un sentiment de « ne rien pouvoir y faire », par exemple avec des élèves qui refusent catégoriquement de participer, ou des élèves à besoins particuliers devant lesquels les EN se trouvent souvent démunis, des élèves qui sont en échec répété, également dans des situations imprévues, en cas d'accident, enfin face à des actions non motivées par le travail scolaire ou de transgression de règles et que les enseignants disent se sentir incapables d'endiguer. Ce sentiment d'impuissance est parfois associé un second sentiment : le manque de reconnaissance de la part de l'équipe (décalages avec l'équipe des professionnels, 5 situations, 1,8\% du total) ou de la part des élèves (manque de complicité entre l'EN et les élèves, 8 situations, 2,9\% du total). Cette absence de reconnaissance est sous-jacente dans la quasi-totalité des situations négatives. Ainsi, les situations où les élèves transgressent $(28,1 \%)$ ou refusent de pratiquer $(3,6 \%)$ sont présentées non seulement comme des situations où l'EN se sent incapable d'infléchir le cours des événements mais aussi comme des écarts au « contrat pédagogique de base » qui lui dénient en quelque sorte le rôle et le statut qui lui sont dévolus (Filloux, 1978).

Cette interprétation fait écho aux principales conclusions de la littérature relative à la problématique de la construction identitaire des EN (Beijaard, Meijer \& Verloop, 2004 ; Sutherland, Howard \& Markauskaite, 2010 ; Méard \& Zimmermann, 2014 ; Nichols, Schutz, Rodgers \& Silica, 2017) qui pointent l'importance dans le processus de ces deux dimensions : le sentiment de pouvoir agir et la reconnaissance (agentivity, recognition). Dans nos résultats, cette lecture trouve sa confirmation selon nous dans les trois seules situations à propos desquelles les EN déclarent qu'elles ont eu un effet négatif sur leur développement professionnel. Dans le premier cas, le tuteur demande à l'EN de réaliser à l'improviste auprès des élèves une activité qui dégénère. Celui-ci se sent décrédibilisé face aux élèves et impuissant face à ce qu'il juge une injustice de la part d'un collègue. Dans le deuxième cas, l'EN se rend compte qu'une de ses élèves a été lourdement sanctionnée au cours d'un conseil des maîtres auquel il n'a pas été convié, donc sans qu'il ait été consulté. Dans le troisième cas, une élève en classe déclare à haute voix et à plusieurs reprises 


\section{eJRIEPS 45 (Avril 2019)}

«c'est nul votre exercice », « on a déjà fait ça 1000 fois ». Dans les trois situations, le sentiment d'impuissance est exprimé explicitement par l'EN, associé à une dévalorisation patente du statut d'enseignant, une détérioration de sa reconnaissance professionnelle.

A l'opposé, l'ensemble des situations associées à des émotions positives dégage une impression de dépassement des difficultés et d'atteinte des objectifs spécifiques de l'enseignant : par exemple, motiver les élèves, faire apprendre les élèves, même ceux qui doivent bénéficier de mesures spécifiques, et réussir à les faire progresser. On semble toucher ici le cœur du métier, et le sentiment qui émerge de ces situations pour l'EN est majoritairement qu'il peut «influencer l'apprentissage des élèves en leur transmettant le plaisir de la réussite » (par exemple dans la situation 111a). Ce sentiment selon lequel «ce qui s'est passé dépend de moi », ce sentiment de "pouvoir y faire quelque chose », de pouvoir agir, est souvent associé au fait que l'action réalisée et ses conséquences sont publics, sous le regard des autres, ce qui entraîne aussi un sentiment d'être " reconnu » professionnellement (Nichols et al., 2017), parfois même explicitement par les collègues et par les élèves. Ceci incite à penser que la reconnaissance et le manque de reconnaissance sont en lien avec la fragile construction d'identité professionnelle des enseignants durant leurs premières années d'enseignement, certains se sentant inclus, d'autres exclus du collectif de travail de leur établissement scolaire (Méard \& Zimmermann, 2014). Les défis ne semblent donc pas uniquement en rapport avec les problèmes disciplinaires au sein de la classe (Veenman, 1984), les différences individuelles entre les élèves, la charge de travail ou encore la pression du métier sur les EN (Kyriacou \& Kunc, 2007), mais aussi avec la place que se fait l'enseignant en début de carrière, au sein d'un établissement scolaire. C'est ce qu'illustre par exemple l'étude de Montagne (2011) à propos d'une EN en contexte difficile. Celle-ci, après avoir énoncé explicitement à propos d'élèves contestant sa place d'enseignante « ce qui m'a choquée, c'était leur attitude envers moi. Ils m’ont ignorée : pour eux, je n'existais pas » (ibid., p.522), est parvenue à rétablir la situation.

La présence de ces quatre sentiments antagonistes exprimés sous forme écrite, par les 139 EN (se sentir / ne pas se sentir capable d'agir ; se sentir / ne pas se sentir reconnu) permet selon nous d'intégrer la compréhension des situations émotionnellement marquantes pour les EN en EPS dans une perspective qui embrasse un processus plus large de développement professionnel.

\section{Conclusion}




\section{eJRIEPS 45 (Avril 2019)}

L'étude présentée ici est un arrêt sur image des situations émotionnellement marquantes vécues par les EN en EPS en Suisse romande, situations analysées à partir d'une typification construite ad hoc. Trois limites principales nous poussent à envisager des perspectives futures à ce travail empirique. D'abord, les résultats obtenus devraient être complétés par des travaux similaires dans d'autres contextes et dans d'autres matières scolaires. En effet, certains types de situations émotionnellement marquantes comportant des problèmes de sécurité ou des transgressions des règles sont particulièrement prégnantes en EPS.

De plus, les types construits par la grounded theory, malgré le nombre important de données et les précautions de deux chercheurs sur plusieurs mois, mériteraient d'être soumis à des expérimentations plus systématiques pour vérifier leur fidélité et leur pertinence afin que, à terme, un traitement quantitatif puisse être réalisé.

Enfin, cette étude ne répond que partiellement à la question de la contribution des émotions au développement professionnel d'EN en EPS. Pourquoi, suite à des expériences émotionnelles fortes, certains enseignants débutants abandonnent le métier et pourquoi d'autres se développent? Seule une étude longitudinale de type clinique de plusieurs mois, voire d'une année, avec plusieurs EN, permettrait à notre avis d'analyser le processus développemental éventuel à la suite de situations émotionnellement marquantes. II serait aussi possible, dans le prolongement de ce travail, de mesurer l'évolution des situations qui affectent le même EN ainsi que l'évolution des émotions ressenties au cours des mois. Ce travail contribuerait à mieux comprendre la nature des discussions et des silences, des demandes d'aides et des repliements dans ce processus, ainsi que la nature des accompagnements à mettre en œuvre dans le but d'améliorer la formation par alternance des enseignants débutants (Descoeudres \& Méard, à paraître) et réduire le phénomène de décrochage professionnel.

\section{Bibliographie}

Aktop, A. \& Beyazgül, G. (2014). Pre-service physical education teacher's attitudes towards teaching Professionals. Procedia - Social and Behavioral Sciences, 116, 3194-3197.

Avalos, B. \& Aylwin, P. (2007). How young teachers experience their professional work in Chile. Teaching and Teacher Education, 23, 515-528.

Ayme, S., Ferrand, C. \& Cogerino, G. (2011). Comportements déviants des élèves et émotion de colère des enseignants d'EPS. Carrefours de l'Education, 32, 213-227. 


\section{eJRIEPS 45 (Avril 2019)}

Becker, E. S., Goetz, T., Morger, V., \& Ranellucci, J. (2014). The importance of teachers' emotions and instructional behavior for their students' emotions - An experience sampling analysis. Teaching and Teacher Education, 43, 15-26.

Beijaard, D., Meijer, P.-C., Verloop, N. (2004). Reconsidering research on teachers' professional identity. Teaching and Teacher Education, 20, 107-128.

Brackett, M. A., Floman, J. L., Ashton-James, C., Cherkasskiy, L., \& Salovey, P. (2013). The influence of teacher emotion on grading practices: a preliminary look at the evaluation of student writing. Teachers and Teaching: Theory and Practice, 19(6), 634-646.

Bullough, R. V. (2009). Seeking Eudaimonia: The emotions in learning to teach and to mentor. In P. A. Schutz, \& M. S. Zembylas (Eds.), Advances in teacher emotion research (pp.33-53). Dordrecht: Springer.

Burel, N. (2014). L'ancrage des compétences émotionnelles dans le corps vivant : études auprès d'enseignants d'EPS. Recherches \& Educations, 12, 89-103.

Burke, J., Schuck, S., Aubusson, P., Buchanan, J., Louvière, J. J., \& Prescott, A. (2013). Why do early career teachers choose to remain in the profession? The use of bestworse scaling to quantify key factors. International Journal of Educational Research, 62, 259-268.

Chang, M. L. (2013). Toward a theoretical model to understand teacher emotions and teacher burnout in the context of student misbehavior: appraisal, regulation and coping. Motivation and Emotion, 37, 799-817.

Chen, J. (2016). Understanding teacher emotions: The development of a teacher emotion inventory. Teaching and Teacher Education, 55, 68-77.

Christensen, E. (2013). Micropolitical staffroom stories: Beginning health and physical education teachers' experience of the staffroom. Teaching and Teacher Education, 30, 74-83.

Clot, Y. (2017). Travail et pouvoir d'agir. Paris: Presses Universitaires de France/Le Travail humain.

Colakoglua, F. \& Yılmaz, T. (2014). Burnout levels of physical education teachers according to personal Factors. Procedia - Social and Behavioral Sciences, 152, 409414.

Cowie, N. (2011). Emotions that experienced English as a Foreign Language (EFL) teachers feel about their students, their colleagues and their work. Teaching and Teacher Education, 1(27), 235-242. 


\section{eJRIEPS 45 (Avril 2019)}

Craig, C.J. (2014). From stories of staying to stories of leaving: A US beginning teacher's experience. Journal of Curriculum Studies, 46, 81-115.

Craig, C.J. (2017). International teacher attrition: multiperspective views. Teachers and Teaching: theory and practice, 23(8), 859-862.

Cowie, N. (2011). Emotions that experienced English as a Foreign Language (EFL) teachers feel about their students, their colleagues and their work. Teaching and Teacher Education, 1(27), 235-242.

Damasio, A. (1994). Descartes' error: Emotion, reason and the human brain. New York: Grosset/Pulman.

De Mauro A.A \& Jennings, P.A. (2016). Pre-service teachers' efficacy beliefs and emotional states. Emotional and Behavioural Difficulties, 21(1), 119-132.

Derri, V., Papamitrou, E., Vernadakis, N., Koufou, N. \& Zetou, E. (2014). Early professional development of physical education teachers: Effects on lesson planning. Procedia Social and Behavioral Sciences, 152, 778-783.

Descoeudres, M. \& Méard, J. (à paraître). La dimension émotionnelle du développement professionnel de l'enseignant débutant dans la formation en alternance: revue de questions. Les Dossiers des Sciences de l'Education.

Filloux, J. (1978). Du contrat pédagogique. Le discours inconscient de l'école. Paris: Dunod.

Glaser B. \& Strauss A.L. (1978). The Discovery of Grounded Theory: Strategies for Qualitative Research. New York: Adline de Gruyter.

Gore, J. M., \& Bowe, J. M. (2015). Interrupting attrition? Re-shaping the transition from preservice to inservice teaching through quality teaching rounds. International Journal of Educational Research, 73, 77-88.

Hagenauer, G., \& Volet, S. E. (2014). I don't hide my feelings, even though I try to: insight into teacher educator emotion display. Australian Educational Researcher, 41, 261281.

Harfitt, G. J. (2015). From attrition to retention: A narrative inquiry of why beginning teachers leave and then rejoin their profession. Asia Pacific Journal of Teacher Education, 43(1), 22-35.

Hargreaves, A. (1998). The emotional practice of teaching. Teaching and Teacher Education, 14(8), 835-854.

Hargreaves, A. (2000). Mixed emotions: teachers' perceptions of their interactions with students, Teaching and Teacher Education, 16(8), 811-826. 


\section{eJRIEPS 45 (Avril 2019)}

Hong, J. Y. (2010). Pre-service and beginning teachers' professional identity and its relation to dropping out the profession. Teaching and Teacher Education, 26, 1530-1543.

Jeu, B. (1977, rééd.1984). Le sport, l'émotion, l'espace. Paris : Vigot.

Johnson, B., Down, B., Le Cornu, R., Peters, J., Sullivan, A., Pearce, J. \& Hunter, J. (2014). Promoting early career teacher resilience: A framework for understanding and acting. Teachers and Teaching: Theory and Practice, 20, 530-546.

Jokikokko, K., Uitto, M., Deketelaere, A. \& Estola, E. (2017). A beginning teacher in emotionnaly intensive micropolitical situations. International Journal of Educational Research, 81, 61-70.

Kim, H., \& Y. Cho (2014). Pre-Service Teachers' Motivation, Sense of Teaching Efficacy, and Expectation of Reality Shock. Asia-Pacific Journal of Teacher Education 42(1), 67-81.

Kyriacou, C., \& Kunc, R. (2007). Beginning teachers' expectations of teaching. Teaching and Teacher Education, 23, 1246-1257.

Lanthaume, F. \& Hélou, C. (2008). La souffrance des enseignants. Paris : PUF.

Lassila, E \& Uitto, M. (2016). The tensions between the ideal and experienced: teacherstudent relationships in stories told by beginning Japanese teachers. Pedagogy, Culture \& Society, 24(2), 205-219.

Le Maistre, C., and A. Paré (2010). Whatever It Takes: How Beginning Teachers Learn to Survive. Teaching and Teacher Education, 26, 559-564.

Malo, A. (2008). Le stagiaire comme praticien réflexif : un point de vue constructiviste et non déficitaire du développement du savoir professionnel en enseignement. In E. Correa Molina \& C. Gervais (Eds). Les stages en formation à l'enseignement : pratiques et perspectives théoriques (pp.103-124). Québec : Presses de l'Université du Québec.

Méard, J. (2013). L'activité des élèves en risque de décrochage scolaire en EPS : la dynamique des interactions en classe. eJRIEPS, 30, 99-104.

Méard, J. \& Zimmermann, P. (2014). La construction identitaire des enseignants débutants et l'émancipation par rapport à la prescription. Travail et apprentissages. 12, 65-78.

Montagne, Y. (2011). «Savoir-y-faire » avec les élèves ; repérer une compétence professorale d'énonciation par une étude de cas. Revue des sciences de l'éducation, 37(3), 507-541.

Moussay, S., Malo, S. \& Méard, J. (2012). Apprendre le métier d'enseignant face à des élèves et avec des formateurs : l'apport d'une pluralité de regards. Recherche et formation, 69, 135-150. 


\section{eJRIEPS 45 (Avril 2019)}

Nichols, S.L., Schutz, P.A., Rodgers K. \& Silica, K. (2017). Early career teachers' emotion and emerging teacher identities. Teachers and Teaching: theory and practice, 23(4), 406-421.

Ottet, F. \& Méard, J. (2018). Les liens entre engagement corporel et développement professionnel de l'enseignant-e d'éducation physique novice : étude exploratoire. Revue Suisse des Sciences de l'Education, 40(1), www.rsse.ch

Park, S., Oliver, J. S., Johnson, T. S., Graham, P., \& Oppong, N. K. (2007). Colleagues' roles in the professional development of teachers: results from a research study of national board certification. Teaching and Teacher Education: An International Journal of Research and Studies, 23(4), 368-389.

Parrott, W.G. (2001). Emotions in Social Psychology: Essential Readings. Key readings in social psychology. Hove: Psychology Press.

Petiot, O., Visioli, J. \& Desbiens, J.F. (2015). Perceptions d'enseignants du secondaire concernant leurs inducteurs émotionnels en situation de classe. Revue française de pédagogie, 193, 41-56.

Pillen, M., Beijaard, D. \& den Brok, P. (2013). Professional identity tensions of beginning teachers. Teachers and Teaching, 19(6), 660-678.

Ria, L. (2001). Les préoccupations des enseignants débutants en Education Physique et Sportive. Thèse de Doctorat. Université Montpellier I (non publiée).

Ria, L. \& Chaliès, S. (2003). Dynamique émotionnelle et activité : le cas des enseignants débutants. Recherche et Formation, 42, 7-19.

Ria, L., Sève, C., Saury, J., Theureau, J. \& Durand, M. (2003). Beginning teachers' situated emotions: A study of first classroom experiences. Journal of Education for Teaching, 29(3), 219-234.

Ria, L., \& Leblanc, S. (2012). Professionnalisation assistée par vidéo : les effets d'une navigation sur NéoPass@ction. Recherches \& éducations, 7, 99-114.

Ria, L., Leblanc, S., Serres, G., \& Durand, M. (2006). Recherche et formation en « analyse des pratiques » : un exemple d'articulation. Recherche et Formation, 51, 43-56.

Ria. L., \& Lussi Borer, V. (2015). Laboratoire d'analyse vidéo de l'activité enseignante au sein des établissements scolaires : enjeux, méthodes et effets sur la formation des enseignants. In Ria, L. (Ed.), Former les enseignants au 21ème siècle. Volume 1 : Etablissement formateur et vidéoformation (pp. 101-117). Bruxelles : De Boeck.

Salaveraa, C., Antoñanzasb, J. L., Noéb, R. \& Teruelb, P (2014). Emotion and anxiety in teachers. Research of Teaching Physical Education. Procedia - Social and 


\section{eJRIEPS 45 (Avril 2019)}

Behavioral Sciences, 132, 577-581.

Saunders, R. (2013). The role of teacher emotions in change: Experiences, patterns and implications for professional development, Journal of Educational Change, 14, 303333.

Schutz, P. A. (2014). Inquiry on teachers' emotion. Educational Psychologist, 49(1), 1-12.

Shoffner, M. (2011). Considering the first year: Reflection as a means to address beginning teachers' concerns. Teachers and Teaching: Theory and Practice, 17, 417-433.

Strauss, A.L., Corbin, J. (1990). Basics of Qualitative Research: Grounded theory Procedures and Techniques. Newbury Park: Sage.

Sutherland L., Howard S., Markauskaite L. (2010). Professional identity creation: Examining the development of beginning preservice teachers' understanding of their work as teachers. Teaching and Teacher Education, 26, 455-465.

Veenman, S. (1984). Perceived Problems of Beginning Teachers. Review of Educational Research, 54(2), 143.178.

Visioli, J. \& Ria, L. (2010). L'expertise des enseignants d'EPS. Quelle prise en compte du contexte des émotions? Movement \& Sport Sciences, 3(71), 3-19.

Visioli, J., Petiot, O. \& Ria, L. (2014). Le jeu émotionnel des enseignants experts en cours d'EPS : un moyen de favoriser le plaisir d'enseigner? Movement \& Sport Sciences, 2(88), 21-34.

Visioli, J., Petiot, O. \& Ria, L. (2015). Vers une conception sociale des émotions des enseignants ? Carrefours de l'éducation, 40, 201-230.

Visioli, J. \& Petiot, O. (2017). La dynamique émotionnelle des enseignants experts en cours d'EPS : quelle relation avec la disponibilité auprès des élèves. eJIRIEPS, 40, 3163.

Vygotski, L. S. (1931/2014). Histoire du développement des fonctions psychiques supérieures. Paris : La dispute.

Vygotski, L. S. (1931-1998). Théorie des émotions. Etude historico psychologique. Paris : L'Harmattan.

Zimmermann P. \& Méard, J. (2016). L'accrochage professionnel des enseignants novices face à des élèves en situation de décrochage scolaire. Education et Formation. 304(2), 73-82. 